Electronic postprint of an article in

Algebra Universalis

Volume 59, Number 3-4, December 2008, Pages 259-275

DOI $10.1007 / \mathrm{s} 00012-008-2025-9$

Article electronically published on November 14, 2008

\title{
The equational compatibility problem for the real line
}

\author{
George F. McNulty \\ In Celebration of the Accomplishments of George A. Grätzer and E. T. Schmidt
}

\begin{abstract}
Walter Taylor proved recently that there is no algorithm for deciding of a finite set of equations whether it is topologically compatible with the real line in the sense that it has a model with universe $\mathbb{R}$ and with basic operations which are all continuous with respect to the usual topology of the real line. Taylor's account used operation symbols suitable for the theory of rings with unit together with three unary operation symbols intended to name trigonometric functions supplemented finally by a countably infinite list of constant symbols. We refine Taylor's work to apply to single equations using operation symbols for the theory of rings with unit supplemented by two unary operation symbols and at most one additional constant symbol.
\end{abstract}

\section{Introduction}

Let $\mathbb{T}$ be a topological space and let $\Gamma$ be a set of equations. We will say that $\Gamma$ is compatible with $\mathbb{T}$ if there is an algebra $\mathbf{T}$ with universe $\mathbb{T}$ whose basic operations are continuous such that $\Gamma$ is true in $\mathbf{T}$. We say that an equation $s \approx t$ is compatible with $\mathbb{T}$ when $\{s \approx t\}$ is. By a signature we mean a system of operation symbols each equipped with a natural number to specify its rank (i.e. its number of arguments). Thus a signature is a function that assigns ranks to operation symbols. Let $\rho$ be a countable computable signature.

The Equational Compatibility Problem for $\mathbb{T}$ and $\rho$ Is there an algorithm for determining of any finite set $\Gamma$ of equations of signature $\rho$ whether $\Gamma$ is compatible with $\mathbb{T}$ ?

This problem is algorithmically unsolvable if no such algorithm exists.

Walter Taylor proved in [18] that the equational compatibility problem is algorithmically unsolvable for the real line and the signature of ring theory enhanced by three additional operation symbols of rank 1 and a countably infinite number of constant symbols. In fact, Taylor devised a method for establishing the algorithmic unsolvability of equational compatibility problems. His method has two parts: a

Presented by R. McKenzie.

Received May 23, 2006; accepted in final form August 29, 2006.

2000 Mathematics Subject Classification: Primary: 08B05; Secondary: 03D35, 22A30, 26B40.

Key words and phrases: Equational compatibility, Hilbert's Tenth Problem, algorithmic unsovability, topological algebra.

(C)2008 Birkhäuser Verlag, Basel 
topological finite determination theorem and the algorithmic unsolvability of an appropriate variant of Hilbert's $10^{\text {th }}$ Problem. Let $\mathbb{T}$ be a topological space, $\rho$ be a signature, and let $\mathbf{T}$ be an algebra of signature $\rho$ with universe $\mathbb{T}$ for which all the basic operations are continuous. The topological algebra $\mathbf{T}$ is finitely determined provided there is a finite set $\Sigma$ of equations of signature $\rho$ which is compatible with $\mathbf{T}$ and up to isomorphism (simultaneously algebraic and topological) $\mathbf{T}$ is the only topological algebra of signature $\rho$ with universe $\mathbb{T}$ which is a topological model of $\Sigma$. We also say that $\mathbf{T}$ is determined by $\Sigma$.

For the second element of Taylor's method we formulate:

Hilbert's $10^{\text {TH }}$ Problem In $n$ VARIABles for The Algebra T

Is there an algorithm for determining of any finite set $\Gamma$ of equations in no more than $n$ variables whether $\Gamma$ has a solution in $\mathbf{T}$ ?

This problem is algorithmically unsolvable if no such algorithm exists.

Taylor's method is predicated on establishing for a given topological algebra $\mathbf{T}$ of signature $\rho$ that it is determined by some finite set $\Sigma$ of equations and that Hilbert's $10^{\text {th }}$ Problem in $n$ variables for $\mathbf{T}$ is not solvable algorithmically. Then the algorithmic unsolvability of the Equational Compatibility Problem for $\mathbb{T}$ and a certain signature $\rho^{\prime}$ can be deduced from these two results as follows. Expand the signature $\rho$ by $n$ new constant symbols $\mathbf{c}_{\mathbf{0}}, \mathbf{c}_{\mathbf{1}}, \ldots, \mathbf{c}_{\mathbf{n}-\mathbf{1}}$ to obtain the signature $\rho^{\prime}$ and for each finite set $\Gamma$ of equations of signature $\rho$ in the variables $x_{0}, x_{1}, \ldots, x_{n-1}$ associate the set $\Gamma^{\prime}$ of equations by substituting the constant symbol $\mathbf{c}_{i}$ for the variable $x_{i}$, for each $i<n$. Then

$\Gamma$ has a solution in $\mathbf{T}$ if and only if $\Sigma \cup \Gamma^{\prime}$ is compatible with $\mathbb{T}$.

This reduces Hilbert's $10^{\text {th }}$ Problem in $n$ variables for the algebra $\mathbf{T}$ to the equational compatibility problem for $\mathbb{T}$ and $\rho^{\prime}$.

Taylor carried out this method for the real line using the topological algebra

$$
\left\langle\mathbb{R},+, \cdot,-, 0,1, \sin ^{*}, \cos ^{*}, \lambda\right\rangle
$$

where $\sin ^{*}(\chi):=\sin \left(\frac{\pi}{2} \chi\right)$ and $\cos ^{*}(\chi):=\cos \left(\frac{\pi}{2} \chi\right)$ for all real numbers $\chi$, and where $\lambda$ is a certain function (described more precisely later) which satisfies $(\lambda(\chi))^{2}=$ $\cos ^{*}\left(\sin ^{*}(\chi)\right)$ for all real numbers $\chi$. We reserve $\sigma$ to denote the signature of this algebra and $\sigma^{\prime}$ to denote its expansion by a countably infinite list of additional constant symbols. Taylor set no bound on the number of variables in Hilbert's $10^{\text {th }}$ Problem for this algebra, leading to all those additional constant symbols.

Taylor also considered smoothness conditions more stringent than continuity for the operations. For each natural number $n$, the set $\mathcal{C}^{n}$ consists of those operations on $\mathbb{R}$ for which all partial derivatives of order $n$ exist and are continuous. A set of equations is $\mathcal{C}^{n}$-compatible with the real line provided the set of equations has a model with universe $\mathbb{R}$ all of whose operations belong to $\mathcal{C}^{n}$. Compatibility and $\mathcal{C}^{0}$-compatiblity coincide. More stringent yet, a set of of equations is real analytic compatible with the real line if all the operations can be chosen to be real analytic.

Recall that two collections $\mathcal{A}$ and $\mathcal{B}$ are said to be recursively inseparable provided every collection $\mathcal{C}$ which includes $\mathcal{A}$ and is dijoint from $\mathcal{B}$ must have an algorithmically unsolvable membership problem. As Taylor notes, his algorithmic 
unsolvability results concerning the several variants of the campatibility problem are all corollaries of the theorem below.

Taylor's Inseparability Theorem. Let the signature be $\sigma^{\prime}$. The collection of finite sets of equations which are not compatible with the real line is recursively inseparable from the collection of finite sets of equations which are real analytic compatible with the real line.

Here we refine this result in two ways. First, we are able to simplify the signatures; in particular, we need no more than one additional constant symbol. Second, we can replace compatibility of finite sets of equations by compatibility of single equations. Where Taylor focussed on one topological algebra, we will consider infinitely many. Let

$$
\mathbf{R}_{\mathrm{ra}}:=\left\langle\mathbb{R},+, \cdot,-, 1, \sin ^{*}, \lambda\right\rangle
$$

and for each natural number $n$ let

$$
\mathbf{R}_{n}:=\left\langle\mathbb{R},+, \cdot,-, 1, \sin ^{*},||_{n}\right\rangle
$$

where $|\chi|_{n}=|\chi|^{2 n+1}$ for all real numbers $\chi$. It is known (and easy) that ||$_{n}$ belongs to $\mathcal{C}^{2 n}$ and hence to $\mathcal{C}^{n}$. Taylor proved in [18] that $\lambda$ is real analytic. In the interests of parsimony, we have dropped the operation $\cos ^{*}$ and the constant 0 , which are definable by terms from the remaining operations. All these algebras have a common signature which we will denote by $\tau$. We use $\tau^{\prime}$ to denote the signature obtained by expanding $\tau$ with a single new constant symbol $\mathbf{c}$. Here is our main result.

\section{The Main Inseparability Theorem.}

(a.) Let the signature be $\tau$ and let $n$ be any natural number. The collection of equations which are not compatible with the real line is recursively inseparable from the collection of equations which are $\mathcal{C}^{n}$-compatible with the real line.

(b.) Let the signature be $\tau^{\prime}$. The collection of equations which are not compatible with the real line is recursively inseparable from the collection of equations which are real analytic compatible with the real line.

This theorem is proven in Section 7.

In 1967 George A. Grätzer and Ralph N. McKenzie [1] announced that any finite set of equations which includes the axioms for rings with unit is logically equivalent to a single equation. A version of their proof can be found in [2]. Independently, in 1966 Alfred Tarski announced the same result, see [17]. A version of Tarski's proof can be found in [13]. Each of these methods is constructive: they provide algorithms which, given any finite set $\Sigma$ of equations which includes the axioms for rings with unit, will produce a single equation $s_{\Sigma} \approx t_{\Sigma}$ which is logically equivalent to $\Sigma$. We refer to the logical equivalence of $\Sigma$ with $s_{\Sigma} \approx t_{\Sigma}$ as the one equation collapse. Since the equational axioms for rings with unit hold in each of the algebras displayed above, we are able to replace the consideration of finite sets of equations with the consideration of single equations. 
To reduce the signatures $\sigma$ and $\sigma^{\prime}$ to $\tau$ and $\tau^{\prime}$ we appeal to methods originated by Daniel Richardson in 1968, see [14]. For part (a) of the Main Inseparability Theorem we also modify Taylor's approach, invoking the undecidability of the (one variable) equational theory of $\mathbf{R}_{n}$ rather than the algorithmic unsolvability of Hilbert's $10^{\text {th }}$ Problem.

The following theorems are what we will need to establish the Main Inseparability Theorem.

Theorem 0. Each of the algebras $\mathbf{R}_{r a}$ and $\mathbf{R}_{n}$ for each natural number $n$ is finitely determined.

Section 1 discusses how to adapt Walter Taylor's arguments to establish this theorem.

Theorem 1. For each of the algebras $\mathbf{R}_{r a}$ and $\mathbf{R}_{n}$ for each natural number $n$, there is no algorithm which will determine of any equation in one variable whether it has a solution in the algebra.

Thus, Hilbert's $10^{\text {th }}$ Problem in 1 variable is algorithmically unsolvable for any of the algebras $\mathbf{R}_{n}$ and $\mathbf{R}_{\mathrm{ra}}$, even when restricted to single equations. Hilbert's $10^{\text {th }}$ Problem is discussed in Section 2. Following an overview of the methods originated by Richardson and some adjustments to these methods in Sections 3 and 4, we prove Theorem 1 in Section 5.

Theorem 2. For each natural number $n$ there is no algorithm which will determine of any equation (in one variable) whether it is true in $\mathbf{R}_{n}$.

A proof of this theorem is given in Section 6 .

As corollaries of the Main Inseparability Theorem, we see that each of the following problems is algorithmically unsolvable:

I. To determine of an arbitrary equation of signature $\tau$ whether it is compatible with the real line.

II. (For each natural number $n$ ) To determine of an arbitrary equation of signature $\tau$ whether it is $\mathcal{C}^{n}$-compatible with the real line.

III. To determine of an arbitrary equation of signature $\tau^{\prime}$ whether it is $\mathcal{C}^{\infty}$ compatible with the real line.

IV. To determine of an arbitary equation of signature $\tau^{\prime}$ whether it is real analytic compatible with the real line.

I would like to thank Walter Taylor for his patient reading many earlier versions of this paper and for his helpful advice.

\section{Finite determination: establishing Theorem 0}

To distinguish the operation symbols of a signature from the basic operations of an algebra, we use boldface for the symbols. So the operation symbols of the signature $\tau$ will be

$$
+, \cdot,-, \mathbf{1}, \sin ^{*} \text {, and } \boldsymbol{\lambda} \text { or one of }||_{n} \text {. }
$$


Strictly speaking, we should use only one unary operation symbol rather than providing $\boldsymbol{\lambda}$ and its infinitely many alternatives. We abuse the notation to increase readability.

Let $\Delta$ be the set of equations listed below, which axiomatize the theory of commutative rings with unit.

$$
\begin{array}{rlrl}
x+(y+z) & \approx(x+y)+z & x \cdot(y \cdot z) & \approx(x \cdot y) \cdot z \\
x+y & \approx y+x & x \cdot y & \approx y \cdot x \\
x+(-\mathbf{1}+\mathbf{1}) & \approx x & x \cdot \mathbf{1} & \approx x \\
x+(-x) & \approx-\mathbf{1}+\mathbf{1} & x \cdot(y+z) & \approx x \cdot y+x \cdot z
\end{array}
$$

Let $\Delta_{n}$ be the set of equations listed below.

$$
\begin{array}{rlrl}
\sin ^{*}(x+y)-\sin ^{*}(x-y) & \approx 2 \sin ^{*} y \sin ^{*}(x+1) & \sin ^{*} 1 & \approx 1 \\
\left(\sin ^{*}\left(\sin ^{*}(x)+1\right)\right)^{2 n+1} & \approx\left|\sin ^{*}\left(\sin ^{*}(x)+1\right)\right|_{n} & |x \cdot x|_{n} \approx|-(x \cdot x)|_{n} \\
|x \cdot x|_{n} & \approx(x \cdot x)^{2 n+1} & &
\end{array}
$$

Finally, let $\Delta_{\text {ra }}$ be the set of the equations listed below.

$$
\begin{aligned}
\sin ^{*}(x+y)-\sin ^{*}(x-y) & \approx 2 \sin ^{*} y \sin ^{*}(x+1) & \sin ^{*} 1 & \approx 1 \\
\sin ^{*}\left(1+\sin ^{*}(x)\right) & \approx(\lambda(x))^{2} & \lambda(x+4) & \approx \lambda(x) \\
\lambda(0) & \approx-1 & \lambda(2) & \approx 1
\end{aligned}
$$

In [18], Taylor proved $\Delta$ determines $\langle\mathbb{R},+, \cdot,-, 1\rangle$. By invoking continuity, especially the familiar intermediate value property of continuous real functions of one variable, Taylor is able to take advantage of the characterization of $\mathbb{R}$ as the essentially unique completely ordered field. In principle, Taylor also proved that $\Delta \cup \Delta_{\text {ra }}$ determines $\mathbf{R}_{\mathrm{ra}}$. Taylor chose a different set of equally familiar trigonometrc identities, using both $\sin ^{*}$ and $\cos ^{*}$ in place of the first two equations in $\Delta_{\text {ra }}$. Taylor applies some well-known results from the theory of functional equations, a branch of mathematics with a long history, to conclude that $\sin ^{*}$ denotes a function of the form $\sin (\alpha \chi)$ for all real numbers $\chi$. Indeed, such findings trace back the d'Alembert in the middle of the eighteenth century and to an 1821 treatise of Cauchy. The same can be said of the first two equations we chose for $\Delta_{\text {ra }}$. See the readable 1955 account of Vaughan in [20], where the result is deduced from the integrability of continuous functions using Riemann sums, and from the solution to second order differential with constant coefficients. To determine the value of $\alpha$ as $\frac{\pi}{2}$, Taylor required that $\sin ^{*}\left(1+\sin ^{*} x\right)$ be nonnegative. The third of the equations listed in $\Delta_{\mathrm{ra}}$ and in $\Delta_{n}$ ensure this nonnegativity. The remaining equations in $\Delta_{\mathrm{ra}}$ and in $\Delta_{n}$ are needed to determine the functions $\lambda$ and ||$_{n}$.

In this way, Walter Taylor's reasoning in [18] can be adjusted to prove our Theorem 0 . 


\section{Hilbert's $10^{\text {th }}$ Problem}

Recall

Hilbert's $10^{\mathrm{TH}}$ Problem in $n$ VARIAbles for the Algebra T

Is there an algorithm for determining of any finite set $\Gamma$ of equations in no more than $n$ variables whether $\Gamma$ has a solution in $\mathbf{T}$ ?

As Hilbert originally framed the problem (for the ring of integers), there was no finite bound on the number of variables under consideration. The negative resolution of Hilbert's $10^{\text {th }}$ Problem actually provides such a bound.

A set $A$ of integers is said to be Diophantine provided there are polynomials $p\left(x, y_{0}, \ldots, y_{n-1}\right)$ and $q\left(x, y_{0}, \ldots, y_{n-1}\right)$ with integer coefficients such that

$$
A=\left\{k \mid p\left(k, y_{0}, \ldots, y_{n-1} \approx q\left(k, y_{0}, \ldots, y_{n-1}\right) \text { has a solution in the integers }\right\} .\right.
$$

This notion can be extended to give meaning to an $m$-ary relation or a $k$-ary function on the integers being Diophantine.

In 1950 it was conjectured by Martin Davis [4] (see also [5]) that the Diophantine sets were exactly the sets for which there are algorithms for listing their elements (in no particular order and with repetitions allowed in the list). While this seemed very doubtful at the time, Martin Davis, Hilary Putnam, and Julia Robinson [7] were able to show in 1961 that it was true, provided the operations allowed in building the "polynomials" $p$ and $q$ included the exponential function $2^{x}$. Not only did they prove that the exponential Diophantine sets were exactly the listable sets, but they also proved that if any function exhibiting roughly exponential growth is Diophantine in the ordinary sense then the Diophantine sets and the listable sets coincide. Other notable references are the 1958 paper of Martin Davis and Hilary Putnam [6] and the 1960 abstract of Julia Robinson [15]. In 1970 Yuri Matiyasevich [9] demonstrated that a function with exponential growth related to the Fibonacci sequence was in fact Diophantine. In this way Davis's Conjecture was verified. Since it had been established in 1936 by Alan Turing [19] that there are listable sets which have algorithmically unsolvable membership problems, Hilbert's Tenth Problem is seen to have negative solution in a strong sense. A fully detailed and highly readable exposition of this result and related matters was given in 1993 by Yuri Matiyasevich [12].

Let $H$ be a set of integers whose characteristic function is not algorithmic (that is membership in $H$ cannot be determined algorithmically) but which is nevertheless listable. There is a particular natural number $n$ and specific polynomials $p\left(x, y_{0}, \ldots, y_{n-1}\right)$ and $q\left(x, y_{0} \ldots, y_{n-1}\right)$ such that

$$
H=\left\{k \mid p\left(k, y_{0}, \ldots, y_{n-1}\right) \approx q\left(k, y_{0}, \ldots, y_{n-1}\right) \text { has a solution in the integers }\right\} .
$$

The smallest known value for $n$ is 9 . This was discovered by Matiyasevich. A detailed exposition can be found in [8]. In particular, this means that there is no algorithm for determining of an arbitrary Diophantine equation in no more than 9 variables, whether it is solvable in the integers.

On the other hand, Hilbert's $10^{\text {th }}$ Problem has long been known to be algorthmically solvable, as a result of Tarski's decision method [16], when formulated for 
the ring of real numbers or the ring of complex numbers. The situation arising from the expansion of the ring of reals (or of complex numbers) by various exponential and trigonometric functions has attracted considerable attention. In particular, Matiyasevich [12] has been able to show that Hilbert's $10^{\text {th }}$ Problem in 1 variable for the algebra $\langle\mathbb{R},+, \cdot,-, 0,1, \sin \rangle$ is algorithmically unsolvable. This result of Matiyasevich depends substantially on earlier work of Daniel Richardson [14] in 1968, B. F. Caviness [3] in 1970, and Paul Wang [21] from 1974. Indeed, Wang's result differs from that of Matiyasevich essentially only because Wang needed to include $\pi$ as a constant of the algebra. However, this result is not quite suitable since we must contend with the function $\sin ^{*}$ rather than the function sin. We modify a key argument of Richardson below.

\section{The methods of Richardson, Wang, and Matiyasevich}

Richardson's 1968 paper contains a number of interesting results, among which we draw attention to the three which are directly relevant here.

Richardson's First Theorem. There is a natural number $n$ so that Hilbert's $10^{\text {th }}$ Problem in $n$ variables for $\mathbf{R}^{\dagger}=\langle\mathbb{R},+, \cdot,-, 0,1 \text {, exp, } \sin , \ln 2, \pi, q\rangle_{q \in \mathbb{Q}}$ is algorithmically unsolvable.

Let $v$ be the signature appropriate to the algebra $\mathbf{R}^{\dagger}$ over the reals that occurs in the statement of Richardson's First Theorem.

Richardson's Second Theorem. There is no algorithm which, given a term $t(x)$ of signature $v$ in no more than one variable, will determine if there is a real number $r$ such that $t^{\mathbf{R}^{\dagger}}(r)$ is negative.

Let $\mathbf{R}^{\ddagger}=\langle\mathbb{R},+, \cdot,-, 0,1, \exp , \sin ,||, \ln 2, \pi, q\rangle_{q \in \mathbb{Q}}$. Richardson also proved

Richardson’s Third Theorem. The one-variable equational theory of $\mathbf{R}^{\ddagger}$ is algorithmically undecidable.

Daniel Richardson's work was accomplished before Matiyasevich completed the resolution of Hilbert's $10^{\text {th }}$ Problem. Instead, Richardson used the result of Davis, Putnam, and Robinson [7] that the version of Hilbert's $10^{\text {th }}$ Problem over the integers for exponential Diophantine equations is algorithmically unsolvable. For this reason, Richardson considered an algebra over the reals which included among its basic operations the natural exponential function and, as a distinguished constant, the natural logarithm of 2 . The latter was needed since the Davis-Putnam-Robinson exponential Diophantine equations involved $2^{x}$ and Richardson's method invoked partial derivatives. The other basic operations Richardson demanded over the reals were the ring operations of addition, multiplication, negation, 0 , and 1 , the sine function, $\pi$ as a distinguished constant, and a distinguished constant for each rational. For his Third Theorem he needed the absolute value function as well. A careful reading of Richardson's work [14] shows that all those rationals (apart from 1) need not be distinguished as constants. B. F. Caviness [3], also not yet aware of Matiyasevich's work, points out that in the event that Hilbert's $10^{\text {th }}$ Problem 
is algorithmically unsolvable (as it indeed is), then the exponential function and ln 2 can be dropped as basic operations from Richardson's results. Caviness also simplified some parts of Richardson's argument.

By 1974, the time of Paul Wang's paper [21], the resolution of Hilbert's $10^{\text {th }}$ Problem by Matiyasevich was known. Let

$$
\mathbf{R}^{\star}=\langle\mathbb{R},+, \cdot,-, 0,1, \sin , \pi, q\rangle_{q \in \mathbb{Q}} .
$$

Wang was able to show

Wang's Theorem. Hilbert's $10^{\text {th }}$ Problem in 1 variable for $\mathbf{R}^{\star}$ is algorithmically unsolvable.

In section 9.2 of his book [12] Matiyasevich presents an exposition of a version of these results. In the process he drops the rationals as distinguished constants and, more surprisingly, is able to drop $\pi$ as well, leaving only the ring operations and the sine function. However, it is Wang's Theorem to which we will appeal most directly. Except at one important point, the way in which $\pi$ and the sine function enter into Richardson's original argument, and into its subsequent adaptations by Caviness and Wang, is in expressions like

$F_{p}\left(x_{0}, \ldots, x_{n-1}\right)=(n+1)^{2}\left[p^{2}\left(x_{0}, \ldots, x_{n-1}\right)+\sum_{i<n}\left(\sin ^{2} \pi x_{i}\right) k_{i}\left(x_{0}, \ldots, x_{n-1}\right)\right] \mathbf{- 1}$

where $p\left(x_{0}, \ldots, x_{n-1}\right)$ and $k_{i}\left(x_{0}, \ldots, x_{n-1}\right)$ are certain polynomials with integer coefficients. Such expressions can be easily framed in terms of Taylor's sin* instead of in terms of $\sin$ and $\pi$ :

$F_{p}\left(x_{0}, \ldots, x_{n-1}\right)=(n+1)^{2}\left[p^{2}\left(x_{0}, \ldots, x_{n-1}\right)+\sum_{i<n}\left(\sin ^{*} 2 x_{i}\right)^{2} k_{i}\left(x_{0}, \ldots, x_{n-1}\right)\right] \mathbf{- 1}$

Now Richardson's Second Theorem and Wang's Theorem depend on two lemmas. Here is the first:

The Richardson-Caviness Inequality Lemma. There is an algorithm which given any polynomial $p\left(x_{0}, \ldots, x_{n-1}\right)$ with integer coefficients will produce $n$ polynomials $k_{0}\left(x_{0}, \ldots, x_{n-1}\right), \ldots, k_{n-1}\left(x_{0}, \ldots, x_{n-1}\right)$ also with integer coefficients such that these polynomials exceed 1 when evaluated at any $n$-tuple of reals, and such that the following statements are equivalent:

(i) there is a solution of $p\left(x_{0}, \ldots, x_{n-1}\right) \approx \mathbf{0}$ over the integers;

(ii) there is an n-tuple of real numbers to which the term function denoted by $F_{p}\left(x_{0}, \ldots, x_{n-1}\right)$ over the reals assigns a value less than 0 .

The proofs of this lemma found in $[14,3,21]$ all carry over without significant change when framed in terms of $\sin ^{*}$ in place of $\sin$ and $\pi$. It is interesting to note that these arguments make use of the Mean Value Theorem in Several Variables.

The second lemma is the key to squeezing down to one variable. Call a system $\left\langle e_{0}, e_{1}, e_{2}, \ldots\right\rangle$ of functions on $\mathbb{R}$ a system of approximate decoding functions if and only if given any natural number $m$, any real numbers $\chi_{0}, \ldots, \chi_{m-1}$, and any $\varepsilon>0$, there is a real number $\eta$ so that $\left|e_{i}(\eta)-\chi_{i}\right|<\varepsilon$ for all $i<m$. 
TAYLOR'S ALGORITHMIC UNSOLVABILITY OF TOPOLOGICAL R-COMPATIBILITY 267

Richardson's Approximate Decoding Lemma. Let $h(x)=x \sin x$ and $g(x)=$ $x \sin x^{3}$. Then

$$
\left.\left\langle h\left(g^{i}(x)\right)\right| i \text { is a natural number }\right\rangle=\langle h(x), h(g(x), h(g(g(x)))), \ldots\rangle
$$

is a system of approximate decoding functions.

A very short argument, given by Wang in [21], is needed to deduce Wang's Theorem from the Richardson-Caviness Inequality Lemma and Richardson's Approximate Decoding Lemma. What is needed to prove our Theorem 1 is a modification of Richardson's Approximate Decoding Lemma.

\section{How to make a system of approximate decoding functions}

Approximate $*$-Decoding Lemma. Let $h(x)=x \sin ^{*} x$ and $g(x)=x \sin ^{*} x^{3}$. Then

$$
\left.\left\langle e_{i}(x)\right| i \text { is a natural number }\right\rangle:=\langle h(x), h(g(x), h(g(g(x)))), \ldots\rangle
$$

is a system of approximate decoding functions.

For each $i<n$ we let $\mathbf{e}_{i}(x)$ be the obvious term that denotes the corresponding decoding function $e_{i}$ in the algebra $\mathbf{R}_{\mathrm{ra}}$ and in each of the algebras $\mathbf{R}_{n}$.

Proof. The claim below is key to proving the Approximate $*$-Decoding Lemma. Our proof has been adapted from an argument of Matiyasevich in [12]. Richardson's original argument can also be adapted in roughly the same way.

Claim. Given any real numbers $\chi$ and $\psi$ and any $\varepsilon>0$, there is a real number $\eta$ such that

$$
\begin{aligned}
|h(\eta)-\chi| & <\varepsilon \\
g(\eta) & =\psi .
\end{aligned}
$$

Proof of the Claim. It is harmless to suppose $\varepsilon<1$. Fix an integer $k$ so large that $4 k-1>|\chi|$ and $4 k-2>|\psi|$ and

$$
\frac{6(4 k-1)^{2}}{(2 k+1) \pi+1}>\frac{4}{\varepsilon}
$$

Consider the closed interval $[4 k-1,4 k+1]$. Notice

$$
\begin{aligned}
& \sin ^{*}(4 k-1)=\sin \frac{\pi}{2}(4 k-1)=\sin \left(2 \pi k-\frac{\pi}{2}\right)=\sin \left(-\frac{\pi}{2}\right)=-1 \\
& \sin ^{*}(4 k+1)=\sin \frac{\pi}{2}(4 k+1)=\sin \left(2 \pi k+\frac{\pi}{2}\right)=\sin \left(\frac{\pi}{2}\right)=1
\end{aligned}
$$

So $\sin ^{*}(x)$ maps $[4 k-1,4 k+1]$ onto $[-1,1]$. Hence, the image of the interval $[4 k-1,4 k+1]$ under the map $h(x)$ must include the interval $[-4 k+1,4 k+1]$. This means that there is $\eta_{0}$ in $[4 k-1,4 k+1]$ so that $h\left(\eta_{0}\right)=\chi$, since $4 k-1>|\chi|$ entails that $-4 k+1<\chi<4 k+1$. 
By continuity, $h$ will map any sufficiently small interval about $\eta_{0}$ into the interval about $\chi$ of radius $\varepsilon$. Our argument depends on finding out how small sufficiently small must be. After a bit of reverse engineering, we take

$$
\delta=\frac{\varepsilon}{(2 k+1) \pi+1} .
$$

Let $\nu$ be any element of $\left[\eta_{0}-\delta, \eta_{0}+\delta\right]$. According to the Mean Value Theorem, we pick $\hat{\eta}$ in $\left[\eta_{0}-\delta, \eta_{0}+\delta\right]$ so that $\left|h(\nu)-h\left(\eta_{0}\right)\right| \leq\left|h^{\prime}(\hat{\eta})\right| \delta$.

Now just observe

$$
\begin{aligned}
|h(\nu)-\chi| & =\left|h(\nu)-h\left(\eta_{0}\right)\right| \\
& \leq\left|h^{\prime}(\hat{\eta})\right| \delta \\
& =\left|\sin \left(\frac{\pi}{2} \hat{\eta}\right)+\frac{\pi}{2} \hat{\eta} \cos \left(\frac{\pi}{2} \hat{\eta}\right)\right| \delta \\
& \leq\left(1+\frac{\pi}{2}\left(\eta_{0}+\delta\right)\right) \delta \\
& \leq\left(1+\frac{\pi}{2}\left(4 k+1+\frac{\varepsilon}{(2 k+1) \pi+1}\right)\right) \frac{\varepsilon}{(2 k+1) \pi+1} \\
& \leq\left(1+2 k \pi+\frac{\pi}{2}+\frac{\pi}{(4 k+2) \pi+2}\right) \frac{\varepsilon}{(2 k+1) \pi+1} \\
& <\left(1+2 k \pi+\frac{\pi}{2}+\frac{\pi}{2}\right) \frac{\varepsilon}{(2 k+1) \pi+1} \\
& =(1+2 k \pi+\pi) \frac{\varepsilon}{(2 k+1) \pi+1} \\
& =((2 k+1) \pi+1) \frac{\varepsilon}{(2 k+1) \pi+1}=\varepsilon
\end{aligned}
$$

This means

$$
|h(\nu)-\chi|<\varepsilon
$$

for any $\nu$ in $\left[\eta_{0}-\delta, \eta_{0}+\delta\right]$.

So it remains to find $\eta$ in $\left[\eta_{0}-\delta, \eta_{0}+\delta\right]$ so that $g(\eta)=\psi$. Now the cubing function maps $\left[\eta_{0}-\delta, \eta_{0}+\delta\right]$ onto $\left[\left(\eta_{0}-\delta\right)^{3},\left(\eta_{0}+\delta\right)^{3}\right]$. Also, observe

$$
\begin{aligned}
\left(\eta_{0}+\delta\right)^{3}-\left(\eta_{0}-\delta\right)^{3} & =6 \eta_{0}^{2} \delta+2 \delta^{3} \\
& \geq 6(4 k-1)^{2} \delta \\
& =6(4 k-1)^{2} \frac{\varepsilon}{(2 k+1) \pi+1} \\
& =\frac{6(4 k-1)^{2}}{(2 k+1) \pi+1} \varepsilon \\
& >\frac{4}{\varepsilon} \varepsilon=4
\end{aligned}
$$

Therefore, as $\nu$ ranges over $\left[\eta_{0}-\delta, \eta_{0}+\delta\right]$ we find that $\nu^{3}$ ranges over an interval of length at least 4 . In turn, this means that $\frac{\pi}{2} \nu^{3}$ ranges over an interval of length at least $2 \pi$. Consequently, as $\nu$ ranges over $\left[\eta_{0}-\delta, \eta_{0}+\delta\right]$ we conclude that $\sin ^{*}\left(\nu^{3}\right)=$ $\sin \left(\frac{\pi}{2} \nu^{3}\right)$ takes on all values between -1 and 1 . 
Then $g(\nu)=\nu \sin ^{*}\left(\nu^{3}\right)$ has to take on all values between $\delta-\eta_{0}$ and $\eta_{0}-\delta$. Recall that $4 k-1 \leq \eta_{0}$. Since we have $|\psi|<4 k-2$, we know that $\psi$ will lie between $\delta-\eta_{0}$ and $\eta_{0}-\delta$ and we can pick $\eta$ in $\left[\eta_{0}-\delta, \eta_{0}+\delta\right]$ so that $g(\eta)=\psi$ and $|h(\eta)-\chi|<\varepsilon$, as desired. This completes the proof of the Claim.

Our whole line of reasoning in support of the Approximate $*$-Decoding Lemma can now be concluded by a straightforward induction on the natural number $m$ to the effect that for all reals $\chi_{0}, \ldots, \chi_{m-1}$ and every $\varepsilon>0$, there is $\eta_{m}$ so that

$$
\left|e_{i}\left(\eta_{m}\right)-\chi_{i}\right|<\varepsilon \text { for all } i<m \text {. }
$$

The base step holds vacuously.

Here is the inductive step. Suppose reals $\chi_{0}, \ldots, \chi_{m}$ are given along with $\varepsilon>0$. The inductive hypothesis applied to the system $\chi_{1}, \ldots, \chi_{m}$ yields $\eta_{m}$ so that

$$
\left|e_{i}\left(\eta_{m}\right)-\chi_{i}\right|<\varepsilon \text { for all } i \text { with } 1 \leq i \leq m \text {. }
$$

Use the Claim to obtain $\eta_{m+1}$ so that $\left|h\left(\eta_{m+1}\right)-\chi_{0}\right|<\varepsilon$ and $g\left(\eta_{m+1}\right)=\eta_{m}$.

It follows that

$$
\begin{aligned}
\left|e_{0}\left(\eta_{m+1}\right)-\chi_{0}\right| & =\left|h\left(\eta_{m+1}\right)-\chi_{0}\right|<\varepsilon \\
\left|e_{1}\left(\eta_{m+1}\right)-\chi_{1}\right| & =\left|e_{0}\left(g\left(\eta_{m+1}\right)\right)-\chi_{1}\right|=\left|e_{0}\left(\eta_{m}\right)-\chi_{1}\right|<\varepsilon \\
\left|e_{2}\left(\eta_{m+1}\right)-\chi_{2}\right| & =\left|e_{1}\left(g\left(\eta_{m+1}\right)\right)-\chi_{2}\right|=\left|e_{1}\left(\eta_{m}\right)-\chi_{2}\right|<\varepsilon \\
\vdots & \\
\left|e_{m}\left(\eta_{m+1}\right)-\chi_{m}\right| & =\left|e_{m-1}\left(g\left(\eta_{m+1}\right)\right)-\chi_{m}\right|=\left|e_{m-1}\left(\eta_{m}\right)-\chi_{m}\right|<\varepsilon
\end{aligned}
$$

which is exactly what we need. We have devised a system of approximate decoding functions from just - and $\sin ^{*}$. This completes the proof of the Approximate *Decoding Lemma.

\section{Just one unknown: The proof of Theorem 1}

Theorem 1. For each of the algebras $\mathbf{R}_{r a}$ and $\mathbf{R}_{n}$ for each natural number $n$, there is no algorithm which will determine of any equation in one variable whether it has a solution in the algebra.

Proof. We repeat here Wang's argument, with one small change (as Wang allowed the rational number $\frac{1}{2}$ ). Let $\mathbf{R}$ be any of the algebras mentioned in the statement of the theorem.

With each polynomial $p\left(x_{0}, \ldots, x_{8}\right)$ with integer coefficients we will associate a term $G_{p}(x)$ in signature $\tau$ so that the following are equivalent

(i) $p\left(x_{0}, \ldots, x_{8}\right) \approx \mathbf{0}$ has a solution in the integers

(ii) $G_{p}(x)<\mathbf{0}$ has a solution in the real numbers

(iii) $G_{p}(x) \approx 0$ has a solution in the real numbers. 
Moreover, there will be an algorithm which upon input of $p\left(x_{0}, \ldots, x_{8}\right)$ will output the associated $G_{p}(x)$. In this way Hilbert's $10^{\text {th }}$ Problem in one variable for $\mathbf{R}$ will be reduced to Hilbert's $10^{\text {th }}$ Problem in 9 variables for the ring of integers, and Theorem 1 will be established.

First, let $H_{p}\left(x_{0}, \ldots, x_{8}\right)$ be the term

$$
2(10)^{2}\left[p^{2}\left(x_{0}, \ldots, x_{8}\right)+\sum_{i<n}\left(\sin ^{*} 2 x_{i}\right)^{2} k_{i}\left(x_{0}, \ldots, x_{8}\right)\right] \mathbf{- 1},
$$

where the terms $k_{i}\left(x_{0}, \ldots, x_{8}\right)$ are the ones from the Richardson-Caviness Inequality Lemma.

Notice that $H_{p}\left(x_{0}, \ldots, x_{8}\right)=2 F_{p}\left(x_{0}, \ldots, x_{8}\right)+\mathbf{1}$, where $F_{p}\left(x_{0}, \ldots, x_{8}\right)$ comes from the Richardson-Caviness Inequality Lamma as well.

Finally, put

$$
G_{p}(x)=H_{p}\left(\mathbf{e}_{0}(x), \mathbf{e}_{1}(x), \ldots, \mathbf{e}_{8}(x)\right)=2 F_{p}\left(\mathbf{e}_{0}(x), \ldots, \mathbf{e}_{8}(x)\right)+\mathbf{1} .
$$

Now it follows from the Approximate *-Decoding Lemma by the continuity of $H_{p}$ that

$$
\begin{gathered}
H_{p}\left(b_{0}, \ldots, b_{8}\right)<0 \text { for some } b_{0}, \ldots, b_{8} \in \mathbb{R} \\
\text { if and only if } \\
G_{p}(\eta)=H_{p}\left(e_{0}(\eta), \ldots, e_{8}(\eta)\right)<0 \text { for some } \eta \in \mathbb{R} .
\end{gathered}
$$

A similar equivalence prevails with the inequalities going in the other direction.

First, we argue that (i) imples (ii) implies (iii). Suppose that $a_{0}, \ldots, a_{8}$ are integers so that $p\left(a_{0}, \ldots, a_{8}\right)=0$. Then $F_{p}\left(a_{0}, \ldots, a_{8}\right)=-1$, which implies that $H_{p}\left(a_{0}, \ldots, a_{8}\right)=-1$ as well. Consequently, $G_{p}(\mu)$ is negative for some real number $\mu$. On the other hand it is easy to see that $H_{p}\left(\frac{\pi}{4}, \ldots, \frac{\pi}{4}\right)$ must be positive. So $G_{p}(\nu)$ must be positive for some real number $\nu$, by the Approximate $*$-Decoding Lemma and the continuity of $H_{p}$. By the Intermediate Value Theorem there must be a real number $\eta$ so that $G_{p}(\eta)=0$.

To see that (iii) implies (i), suppose that $G_{p}(\eta)=0$. Then there are real numbers $\beta_{0}, \ldots, \beta_{8}$ so that $0=2 F_{p}\left(\beta_{0}, \ldots, \beta_{8}\right)+1$. This means that $F_{p}\left(\beta_{0}, \ldots, \beta_{8}\right)$ is negative. By the Richardson-Caviness Inequality Lemma, we conclude that $p\left(x_{0}, \ldots, x_{8}\right) \approx \mathbf{0}$ has a solution in the integers.

\section{Just one variable: Proof of Theorem 2}

Theorem 2. For each natural number $n$ there is no algorithm which will determine of any equation (in one variable) whether it is true in $\mathbf{R}_{n}$.

Proof. In Section 5 we noted that there is an algorithm which given any polynomial $p\left(x_{0}, \ldots, x_{8}\right)$ with integer coefficients will produce a term $G_{p}(x)$ in one variable so that

$$
\begin{aligned}
& p\left(x_{0}, \ldots, x_{8}\right) \approx \mathbf{0} \text { has a solution in the integers } \\
& \quad \text { if and only if } \\
& G_{p}(x)<\mathbf{0} \text { has a solution in the real numbers }
\end{aligned}
$$


It is our contention that the one-variable equation $\left|G_{p}(x)\right|_{n} \approx\left(G_{p}(x)\right)^{2 n+1}$ fails in $\mathbf{R}_{n}$ if and only if $p\left(x_{0}, \ldots, x_{8}\right) \approx \mathbf{0}$ has a solution in the integers.

This contention holds since $p\left(x_{0}, \ldots, x_{8}\right) \approx \mathbf{0}$ has a solution in the integers if and only if $G_{p}(x)<\mathbf{0}$ has a solution in the reals if and only if the equation $\left|G_{p}(x)\right|_{n} \approx\left(G_{p}(x)\right)^{2 n+1}$ fails in $\mathbf{R}_{n}$.

So Hilbert's $10^{\text {th }}$ Problem over the integers reduces to the algorithmic decision problem for the one-variable equational theory of $\mathbf{R}_{n}$. Hence that latter problem must be algorithmically undecidable.

\section{Proof of the Main Inseparability Theorem}

\section{The Main Inseparability Theorem.}

(a.) Let the signature be $\tau$ and let $n$ be any natural number. The collection of equations which are not compatible with the real line is recursively inseparable from the collection of equations which are $\mathcal{C}^{n}$-compatible with the real line.

(b.) Let the signature be $\tau^{\prime}$. The collection of equations which are not compatible with the real line is recursively inseparable from the collection of equations which are real analytic compatible with the real line.

There is a well-known method for establishing recursive inseparability results. We will use it to prove the Main Inseparability Theorem. Loosely speaking, the method runs as follows. We say that a set $U$ has an algorithmically unsolvable membership problem when there is no algorithm for determining of an input $u$ whether $u \in U$. The recursive inseparability of two sets $A$ and $B$ follows from the existence of an algorithm $\Phi$ with the following properties:

(i.) If $u \in U$, then $\Phi(u) \in B$.

(ii.) If $u \notin U$, then $\Phi(u) \in A$.

Indeed, suppose that $A \subseteq C$ with $C$ and $B$ disjoint. We see that if $u \in U$, then $\Phi(u) \in B$ and so $\Phi(u) \notin C$. On the other hand, if $u \notin U$, then $\Phi(u) \in A$ and so $\Phi(u) \in C$. This means $u \in U$ if and only if $\Phi(u) \notin C$. Therefore, the membership problem for $U$ reduces to the membership problem for $C$. Since the membership problem for $U$ is algorithmically unsolvable, we see that the membership problem for $C$ must also be algorithmically unsolvable. It follows that $A$ and $B$ are recusively inseparable.

Proof of the Main Inseparability Theorem. For part (a) we take $U$ to be the set of all equations of signature $\tau$ which are true in $\mathbf{R}_{n}$ and in which $x$ is the only variable to occur. We take $A$ to be the set of equations of signature $\tau$ which are not compatible with the real line and we take $B$ to be the set of equations of signature $\tau$ which are $\mathcal{C}^{n}$-compatible with the real line. For each equation $p(x) \approx q(x)$ of signature $\tau$ in which no variable other than $x$ occurs we define $\Phi(p(x) \approx q(x))$ to be $s_{\Sigma} \approx t_{\Sigma}$, where $\Sigma=\Delta \cup \Delta_{n} \cup\{p(x) \approx q(x)\}$. Here is where we invoke the one equation collapse. According to Theorem 2, the set $U$ has an algorithmically unsolvable membership problem. Moreover, condition (i.) of our method holds, since if $p(x) \approx q(x)$ is true in $\mathbf{R}_{n}$, then $\Sigma \cup\{p(x) \approx q(x)\}$ is $\mathcal{C}^{n}$-compatible with the 
real line and the same must hold for $\Phi(p(x) \approx q(x))$ since $\Sigma \cup\{p(x) \approx q(x)\}$ and $\Phi(p(x) \approx q(x))$ have the same models. For condition (ii.) suppose $p(x) \approx q(x)$ fails in $\mathbf{R}_{n}$. Then $\Sigma \cup\{p(x) \approx q(x)\}$ is not compatible with the real line, by Theorem 0 . Since $\Sigma \cup\{p(x) \approx q(x)\}$ and $\Phi(p(x) \approx q(x)$ have the same models, we find that $\Phi(p(x) \approx q(x))$ is not compatible with the real line either. In this way part (a) of our theorem is established.

For part (b) we take $U$ to be the set of all equations of signature $\tau$ which are solvable in $\mathbf{R}_{\mathrm{ra}}$ and in which $x$ is the only variable to occur. We take $A$ to be the set of equations of signature $\tau^{\prime}$ which are not compatible with the real line and we take $B$ to be the set of equations of signature $\tau^{\prime}$ which are real analytic compatible with the real line. For each equation $p(x) \approx q(x)$ of signature $\tau^{\prime}$ in which no variable other than $x$ occurs we define $\Phi(p(x) \approx q(x))$ to be $s_{\Sigma} \approx t_{\Sigma}$, where $\Sigma=\Delta \cup \Delta_{\text {ra }} \cup\{p(\mathbf{c}) \approx q(\mathbf{c})\}$. We have invoked the one equation collapse again. According to Theorem 1 , the set $U$ has an algorithmically unsolvable membership problem. Moreover, condition (i.) of our method holds, since if $p(x) \approx q(x)$ is solvable in $\mathbf{R}_{\mathrm{ra}}$, then $\Sigma \cup\{p(\mathbf{c}) \approx q(\mathbf{c})\}$ is real analytic compatible with the real line and the same must hold for $\Phi(p(x) \approx q(x))$ since $\Sigma \cup\{p(\mathbf{c}) \approx q(\mathbf{c})\}$ and $\Phi(p(x) \approx q(x))$ have the same models. For condition (ii.) suppose $p(x) \approx q(x)$ has no solution in $\mathbf{R}_{\mathrm{ra}}$. Then $\Sigma \cup\{p(\mathbf{c}) \approx q(\mathbf{c})\}$ is not compatible with the real line, by Theorem 0 . Since $\Sigma \cup\{p(\mathbf{c}) \approx q(\mathbf{c})\}$ and $\Phi(p(x) \approx q(x))$ have the same models, we find that $\Phi(p(x) \approx q(x))$ is not compatible with the real line either. In this way part (b) of our theorem is established.

\section{REFERENCES}

[1] George A. Grätzer and Ralph N. McKenzie, Equational spectra and reduction of identities, Notices of the American Mathematical Society, 14 (1967) p. 697.

[2] George A. Grätzer and R. Padmanabhan, R., Symmetric difference in abelian groups, Pacific J. Math., 74 (1978) 339-347.

[3] B. F. Caviness, On canonical forms and simplification. Journal of the ACM, 17 (1970) 385-396.

[4] Martin Davis, Arithmetical problems and recursively enumerable predicates (abstract), Journal of Symbolic Logic, 15 (1950) 77-78.

[5] Martin Davis, Arithmetical problems and recursively enumerable predicates, Journal of Symbolic Logic, 18 (1953) 33-41.

[6] Martin Davis and Hilary Putnam, Reductions of Hilbert's Tenth Problem. Journal of Symbolic Logic, 23 (1958) 183-187.

[7] Martin Davis, Hilary Putnam, and Julia Robinson, The decision problem for exponential Diophantine equations. Annals of Mathematics, Second Series, 74 (1961) 425-436.

[8] James P. Jones, Universal Diophantine equations. Journal of Symbolic Logic, 47 (1982) 549-571.

[9] Yuri V. Matiyasevič, Enumerable sets are Diophantine. Soviet Mathematics Doklady, 11 (1970) 354-358.

[10] Yuri V. Matiyasevič, Diophantine representation of recursively enumerable predicates. In Actes du Congrès International des Mathématiciens (Nice 1970), 1 (1971) 235-238, Gauthier-Villars, Paris.

[11] Yuri V. Matiyasevič, Some purely mathematical results inspired by mathematical logic. In Logic, Foundations of Mathematics, and Computability Theory, vol. 1 of Proceedings of the Fifth International congress of Logic, Methodology and Philosophy of Science, (Robert E. 
Butts and Jaakko Hintikka, eds) (1977) 121-127, D. Reidel Publishing Company, Dordrecht, Holland.

[12] Yuri V. Matiyasevich, Hilbert's Tenth Problem, Foundations of Computing Series, MIT Press, Cambridge, Massachusetts, 1993.

[13] George F. McNulty, Minimum bases for equational theories of groups and rings: The work of Alfred Tarski and Thomas C. Green, Annals of Pure and Applied Logic, 127 (2004) 131-153.

[14] Daniel Richardson, Some undecidable problems involving elementary functions of a real variable. Journal of Symbolic Logic,33 (1968) 514-520.

[15] Julia Robinson, The undecidability of exponential Diophantine equations. Notices of the American Mathematical Society, 7 (1960) p. 75.

[16] Alfred Tarski, A decision method for elementary algebra and geometry, University of California Press, Berkeley and Los Angeles, 1951.

[17] Alfred Tarski, Equational logic and equational theories of algebras, pages 275-288 in Contributions to Mathematical Logic: Proceedings of the Logic Colloquium, Hannover 1966, edited by H. A. Schmidt, K. Schütte, and H. J. Thiele, Studies in Logic and Foundations of Mathematics, North-Holland Publishing Company, Amsterdam, 1968.

[18] Walter Taylor, Equations on real intervals, Algebra Universalis, to appear.

[19] Alan Turing, On computable numbers, with an application to the Entscheidungsproblem, Proceedings of the London Mathematical Society 42 (1936) 230-265.

[20] H. E. Vaughan, Characterizing the sine and cosine, Amer. Math. Monthly, 62 (1955) 707-713.

[21] Paul S. Wang, The undecidability of the existence of zeros of real elementary functions. Journal of the Association of Computing Machines, 21 (1974) 586-589.

Department of Mathematics

University of South Carolina

Columbia, SC 29208

USA

E-mail address: mcnulty@math.sc.edu 